\title{
PReS-FINAL-2067: How does the self-experienced HRQOL work for children with JIA - juvenile idiopathic arthritis in Stockholm?
}

\author{
K Berggren \\ From 20th Pediatric Rheumatology European Society (PReS) Congress \\ Ljubljana, Slovenia. 25-29 September 2013
}

\section{Introduction}

The aim of this study was to examine how children/ teenagers with JIA - Juvenile Idiopathic Arthritis and its subgroups perceived their HRQOL - Health related quality of Life and to see if there were any correlation between HRQOL, self-rated ability of ADL and the amounts of inflamed joints.

\section{Objectives}

The subjects were 50 children/teenagers, 8-18 years (mean 13,7), 37 girls and 13 boys. 26/50 with Juvenile Polyarthritis, and 30/50 was treated with biological drugs.

\section{Methods}

ADL was measured with CHAQ - Childhood Health Assessment Questionnaire. The amount of joints was assessed by doctors. The HRQOL-instrument used was Disabkids, both the Generic version and the Arthritis version. The Generic version contains 37 questions and the Arthritis version 15 questions.

\section{Results}

Compared to other children with a chronic disease, the HRQOL estimates of the studied group is lower. The study showed a moderate correlation $(r=-0.43--0.70)$ between CHAQ and 6 of the subgroups in Disabkids generic and in 1 of 2 subgroups in arthritis - Impact. CHAQ correlated to physical functions but also to mental and social functions. Only a mild correlation was seen between the doctors assessment of active joints $(r=0.31)$. The

Astrid Lindgren Childrens Hospital, Karolinska University Hospital, Stockholm, Sweden 